\title{
Development of an Interactive Program to Study of the Continuation Power Flow
}

\author{
J. H. Canossa, A. Bonini Neto, D. A. Alves, F. F. Putti, L. R. A. Gabriel Filho
}

\begin{abstract}
In this paper an interactive program developed to study of the continuation power flow is presented. Both, the graphical user interface and the power flow program have been developed in the Matlab environment thus providing a good computational performance and a users-friendly teaching software. The interactive program allows the user to edit the input and output data files of the selected power systems as well as to visualize and analyze the results directly on the computer screen. Directly from the system one-line diagram, the graphical interface allows the user change the parameters of the system (bus and lines system data); remove the transmission line clicking directly on it; view the buses voltage and angle profiles and the real and reactive power flows. In order to assess the current operating point condition, the user can also obtain the pre- and post-contingency loading margins by tracing the respective $P-V$ curves. All these characteristics make the developed program recommended for educational purpose.
\end{abstract}

Keywords: Power engineering education; P-V curve; Continuation power flow; Power flow analysis.

\section{INTRODUÇÃO}

$\mathrm{U}$ MA área que tem despertado um crescente interesse por parte dos pesquisadores e concessionárias está relacionada com a visualização das informações (tensões das barras do sistema, fluxos de potência nas linhas de transmissão, perdas de potência ativa e reativa no sistema de transmissão) de sistemas elétricos de potência [1]. Tradicionalmente estas informações eram representadas em relatórios na forma de textos, de dados tabulares, em campos numéricos locados em diagramas unifilares e em painéis mímicos nos quais a variação dinâmica dos dados era mostrada por meio de lâmpadas de cores diferentes. Antes do aparecimento das interfaces gráficas o processamento do fluxo de potência era realizado por intermédio de arquivos de texto tanto para a saída quanto para a entrada de dados (informações retiradas de diagramas elétricos unifilares ou a serem plotadas sobre tais diagramas).

Em [2] foi proposto o desenvolvimento de uma interface gráfica voltada para a resolução do problema de planejamento da expansão das linhas de transmissão. A grande dificuldade

Os autores agradecem ao apoio financeiro da Capes.

J. H. Canossa, Mestre em Engenharia Elétrica, Ilha Solteira - Brasil, email: canossaj@hotmail.com.

A. Bonini Neto, Faculdade de Ciências e Engenharia, UNESP, Tupã Brasil (e-mail: bonini@tupa.unesp.br).

D. A. Alves, Departamento de Engenharia Elétrica, UNESP, Ilha Solteira - Brasil (e-mail: dalves@dee.feis.unesp.br).

F. F. Putti, Faculdade de Ciências e Engenharia, UNESP, Tupã - Brasil. (e-mail: fernandoputti@tupa.unesp.br).

L. R. A. Gabriel Filho, Faculdade de Ciências e Engenharia, UNESP, Tupã - Brasil (e-mail: gabrielfilho@tupa.unesp.br).

Corresponding author: A. Bonini Neto. encontrada por usuários ao interagir com estes programas, motivou o desenvolvimento de uma aplicação com interface gráfica. Já em [3], foi desenvolvido uma interface gráfica capaz de otimizar a utilização de métodos numéricos. A ideia consistiu na utilização de um grupo de painéis, sendo que, cada painel abriga a interface de um determinado método.

Não apenas nessa área, mas em muitas outras a interface gráfica tem demonstrado seu poder de aplicação.

Recentemente, em [4] foi desenvolvido um software utilizando a lógica fuzzy para a comparação da massa corporal de bovinos entre si e identificação dos grupos para abate, e dos que necessitam de alimentação mais intensa, utilizando-se das variáveis "massa" e "altura", e a saída IMCFuzzy.

Vários outros trabalhos envolvendo interface gráfica foram desenvolvidos nos últimos anos. [5-9].

A interface gráfica aqui apresentada tem como intuito, auxiliar e facilitar o aprendizado dos estudantes dos cursos de graduação e de pós-graduação em engenharias, relacionados às disciplinas envolvendo energia, na aquisição de conceitos relacionados com o problema de análise estática de estabilidade de tensão em sistemas elétricos de potência. Está análise pode ser realizada por meio da obtenção do perfil de tensão das barras em função de seu carregamento (curvas P-V, Q-V). Entre outras aplicações, estes perfis são usados para determinar os limites de transferência de potência entre áreas de um sistema, ajustar margens, observar o comportamento das tensões das barras do sistema em análise, e comparar estratégias de planejamento. Estas curvas possibilitam a avaliação das condições de operação do sistema para diferentes carregamentos, sendo recomendadas pelas empresas do setor elétrico nacional [10] e internacional [11], para avaliação das margens de estabilidade de tensão, i.e., para a determinação do maior aumento de carga que um sistema pode suportar a partir de um dado ponto de operação. $\mathrm{O}$ cálculo dessa margem exige, primeiramente, a obtenção do ponto de máximo carregamento (PMC) do sistema.

No método de fluxo de potência continuado (FPC) a obtenção do PMC ocorre por meio da variação automática do valor do parâmetro $(\lambda)$. As dificuldades numéricas são superadas pela adição de equações parametrizadas [12-16]. A reformulação das equações de fluxo de potência (FP) visa remover a singularidade da matriz no PMC e, por conseguinte, eliminar os problemas numéricos que surgem em sua vizinhança. Dessa forma, possibilitam que algoritmos com precisão simples sejam usados tanto na vizinhança quanto no próprio PMC. Essa é a razão principal de sua ampla utilização para o traçado das curvas P-V [15-19]. Outra ideia envolvendo o FPC é a análise de contingências (saída de operação de um ou mais componentes do sistema de forma inesperada, por exemplo, a perda de uma linha de transmissão) [20]. A 
simulação também é realizada por meio da interface gráfica desenvolvida.

Neste contexto, este trabalho apresenta a interface gráfica desenvolvida com o intuito de introduzir os conceitos relacionados com a análise estática da estabilidade de tensão, o fluxo de potência continuado e o traçado das curvas P-V de pré- e pós-contingência de um sistema elétrico de potência. Com isso, espera-se auxiliar, facilitar e principalmente, motivar o aprendizado dos estudantes dos cursos de graduação e de pós-graduação em disciplinas envolvendo energia.

\section{MATERIAIS E MÉTODOS}

\section{Equações do Fluxo de Potência Continuado}

Os métodos de FPC utilizados nesse trabalho foram proposto em [15] e [21]; outras metodologias poderiam ter sido utilizadas [22] e [23]. As Figs. 1 e 2 a seguir apresentam os seus respectivos fluxogramas. Na Fig. 1 apresenta-se o método que usa a técnica de parametrização local proposta em [15], enquanto que na Fig.2 apresenta-se o método proposto em [21], o qual usa a equação de uma reta localizada no plano de coordenadas formada pelas variáveis fator de carregamento $(\lambda)$ e a magnitude da tensão nodal $\left(V_{k}\right)$ de uma barra $k$ qualquer.

\section{Programa Interativo para o Fluxo de Potência Continuado}

O desenvolvimento da interface gráfica foi inicializado em [24], sendo aplicada para a análise de conceitos relacionados com os métodos de fluxo de potência convencional e continuado. Mais recentemente foram incorporadas as novas técnicas de parametrizações propostas em [15] e em [21].

$\mathrm{O}$ ambiente Matlab [25] foi utilizado para desenvolver o software, ou seja, programar a interface gráfica e os programas de fluxo de potência continuado. $O$ elemento básico de informação no Matlab é a matriz, a qual não requer dimensionamento prévio e permite a resolução de muitos problemas numéricos em apenas uma fração do tempo que se gastaria para escrever um programa semelhante em outras linguagens tais como o Fortran, o Basic ou $\mathrm{C}++$. Apesar da existência de outras alternativas atraentes, a escolha foi baseada nas seguintes considerações [25]:

$\checkmark \quad$ É uma linguagem comercial de alto nível e amplamente difundida nos cursos de engenharia;

$\checkmark$ Apresenta uma estrutura de dados na forma de matrizes, sem exigir dimensionamento prévio;

$\checkmark$ Inclui uma vasta seleção de Toolboxes: controle, redes neurais, otimização, simulink;

$\checkmark$ Apresenta a disponibilidade de processos numéricos de alta qualidade, incluindo técnicas de esparsidade;

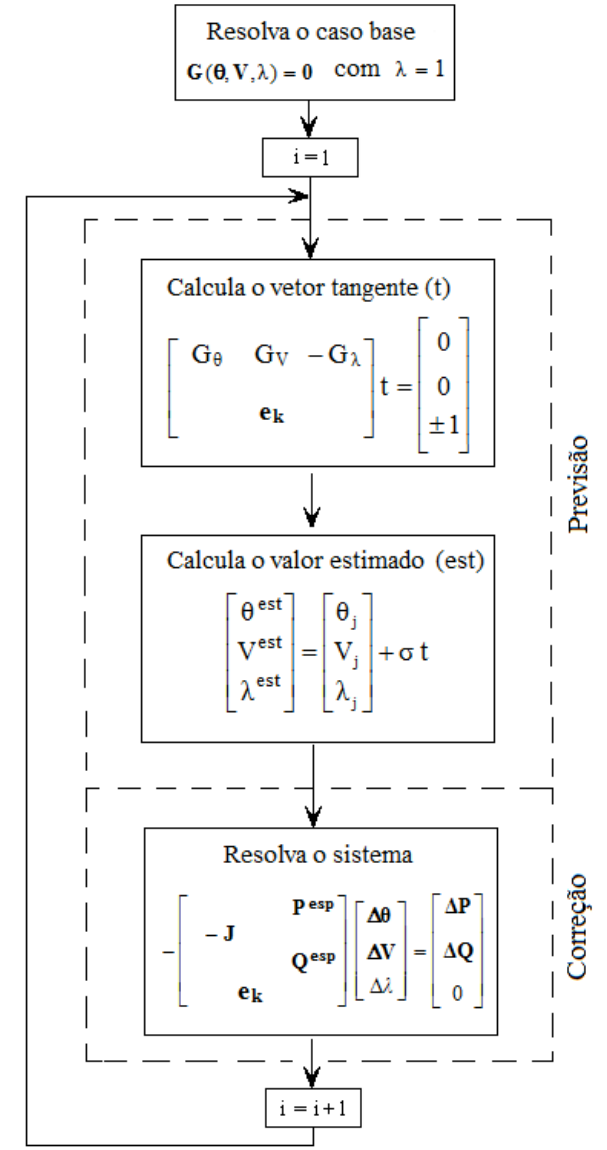

$\mathbf{e}_{\mathbf{k}}=\left[\ldots .0,0, \ldots . ., 1_{k}, \ldots \ldots, 0, \ldots \ldots.\right]$

" $\sigma$ " tamanho do passo preditor

"j” solução atual

Figura 1: Fluxograma do FPC proposto em [15].

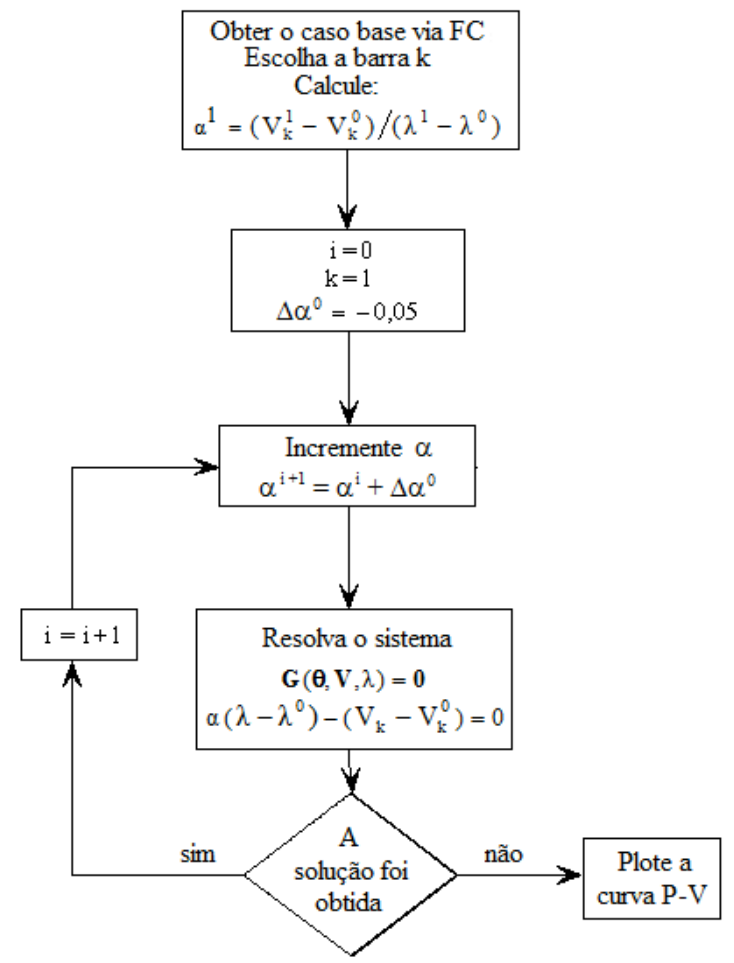

Figura 2: Fluxograma do FPC proposto em [21]. 
$\checkmark$ Apresenta um ambiente de visualização e programação simples;

$\checkmark$ Possui recursos para o desenvolvimento de interfaces gráficas portáveis e poderosas;

$\checkmark$ Permite a criação dinâmica de links para subrotinas em C ou Fortran.

Portanto, o Matlab não só é um software interativo de alta performance voltado para o cálculo numérico e científico, como também integra análise numérica, cálculo com matrizes, processamento de sinais e construção de gráficos num ambiente fácil de usar, onde problemas e soluções podem ser expressos como eles são escritos na matemática ou na forma de uma linguagem de programação. A interface gráfica foi implementada aplicando a função Figure e suas propriedades (Color, Units, MenuBar, NumberTitle, Position, Resize e Name). Também utilizou o comando de controle Uicontrol e suas propriedades: (Units, Position, String, Style, Enable, CallBack, Visible, BackgroundColor, ForegroundColor e HorizontalAlignment) [25].

Pode-se criar uma janela por meio da função Figure e formatar essa janela através de seus parâmetros (alguns destes parâmetros serão mostrados a seguir). A Fig. 3 apresenta uma janela desenvolvida com a função Figure e seus parâmetros devidamente configurados.

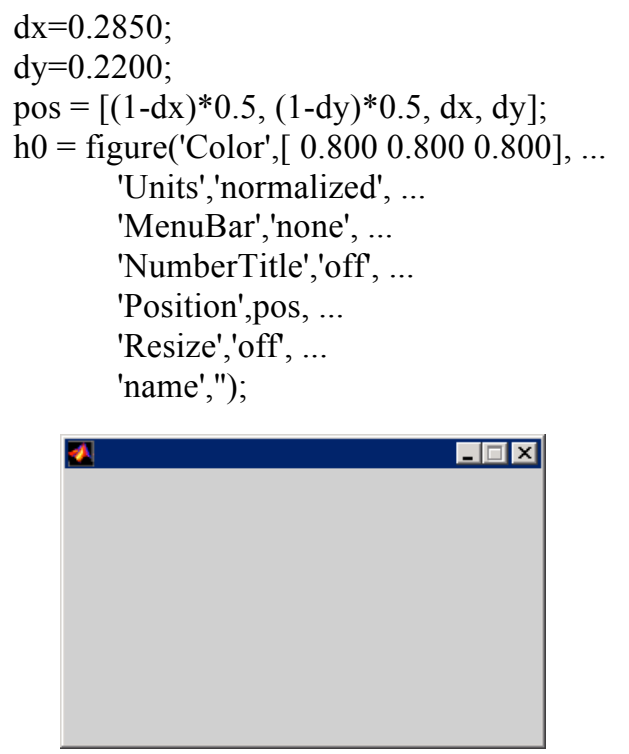

Figura 3: Exemplo da função Figure.

O Uicontrol é um comando de controle para a janela que está ativa. Para criar os controles, devem-se configurar apropriadamente seus parâmetros. Os parâmetros e a Fig. 4 a seguir apresentam o exemplo do comando Uicontrol.

$$
\begin{aligned}
& \text { h1 = uicontrol('Parent',h0, ... } \\
& \text { 'Units','normalized', ... } \\
& \text { 'BackgroundColor',[[ } \left.\begin{array}{lll}
1 & 1 & 1
\end{array}\right], \ldots \\
& \text { 'ForegroundColor',[0.000 } 0.0000 .502], \ldots \\
& \text { 'HorizontalAlignment','center', ... } \\
& \text { 'Position',[0.0787 0.7678 0.8287 0.1547], ... } \\
& \text { 'String','Sistemas:', ... }
\end{aligned}
$$

'FontSize',17,...

'Fontname','Arial',...

'Style','text', ...

'Tag','StaticText1');

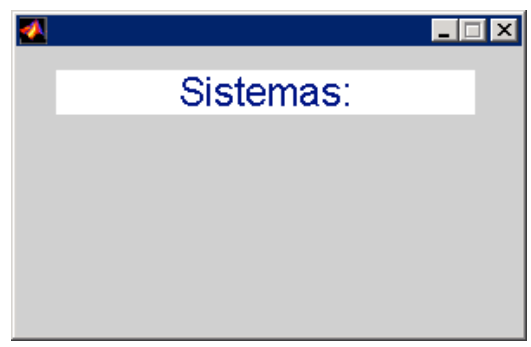

Figura 4: Exemplo do BackgroundColor.

Todos os outros comandos descritos anteriormente e utilizados para desenvolver a interface gráfica presente neste trabalho, podem ser encontrados em [25].

\section{RESULTADOS E DISCUSSÃO}

O sistema teste utilizado foi do IEEE (Institute of Electrical and Electronic Engineers) de 30 barras. O diagrama unifilar do sistema desenvolvido no programa interativo pode ser observado na Fig. 5.

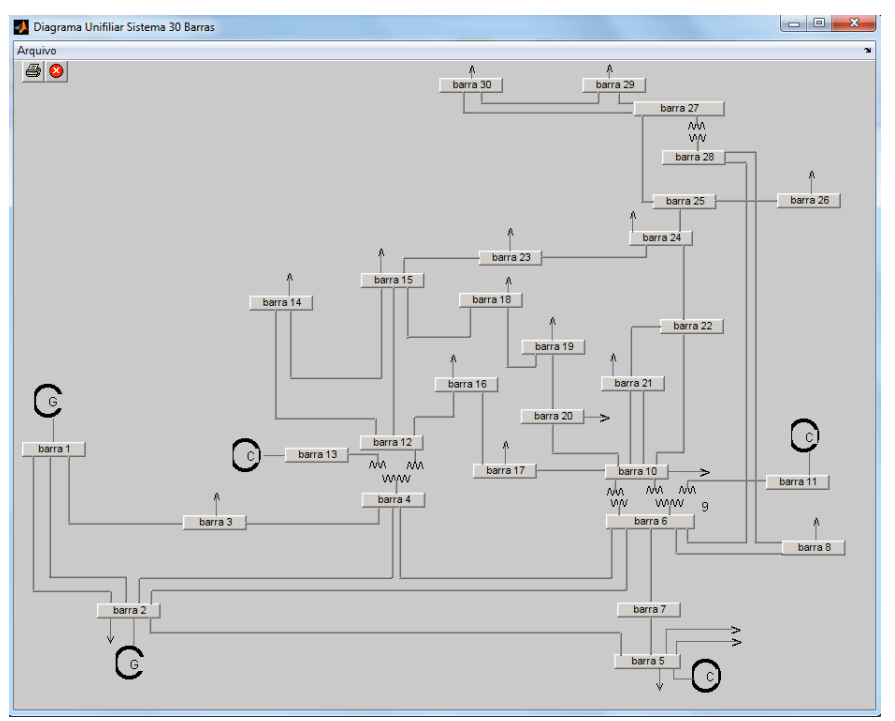

Figura 5 - Diagrama unifilar do sistema IEEE-30 barras.

A janela inicial do programa com a respectiva Barra de Menu discriminada é apresentada na Fig. 6. Escolhendo a opção Arquivo da Barra de Menu ou o ícone Carregar Sistema, uma nova caixa de diálogo denominada "Escolha do Sistema" se abre e o usuário tem a opção de escolher o sistema desejado conforme apresentado na Fig. 7. Pode-se observar que outro sistema poderia ter sido escolhido, como exemplo o sistema apresentado na Fig. 8, caso o usuário escolhesse o sistema IEEE-14 barras. 


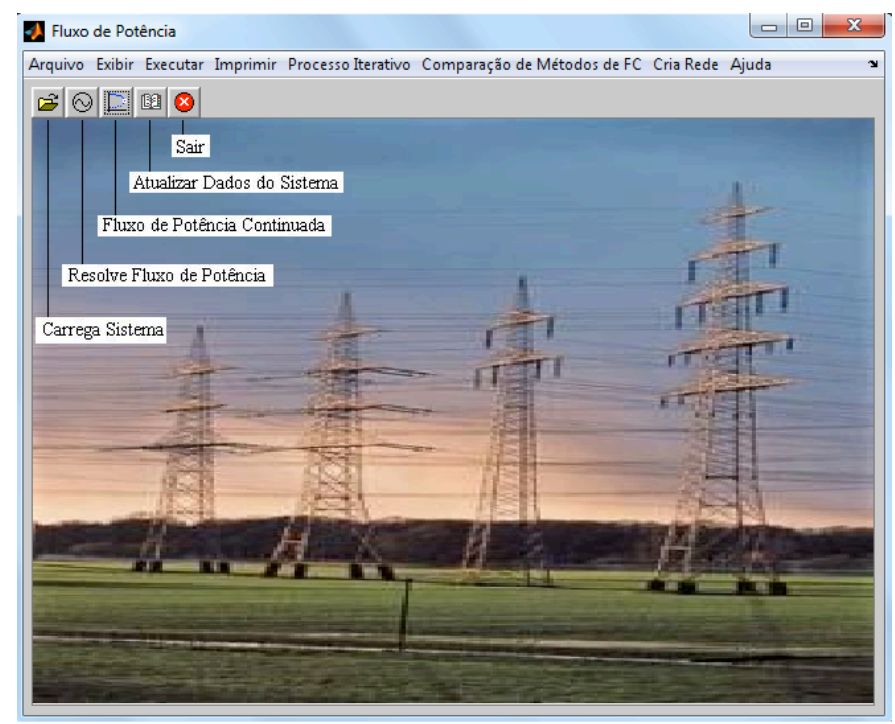

Figura 6- Tela principal do programa Fluxo de Potência.

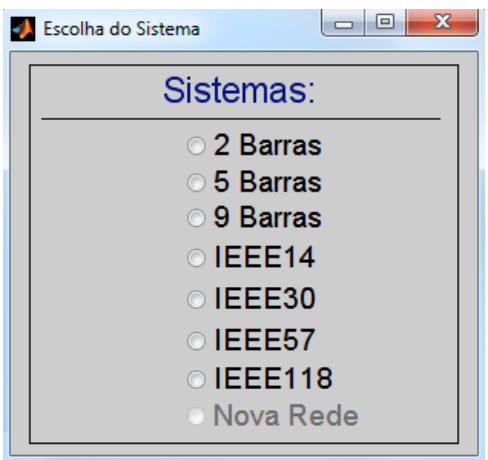

Figura 7 - Caixa de diálogo "Escolha do Sistema" para a escolha do sistema a ser simulado.



Figura 8 - Diagrama unifilar do sistema IEEE-14 barras.

Para sistemas maiores, no caso do sistema IEEE-118 barras, o diagrama unifilar não é apresentado devido ao grande número de barras e linhas de transmissão. Observa-se que até o presente momento a interface foi desenvolvida para fins educacionais, e não para análise de grandes sistemas.

Os métodos do FPC disponibilizados são baseados nos equacionamentos apresentados nos fluxogramas do item II: parametrizado por $\lambda$ ou pela magnitude de tensão de uma barra qualquer $\left(\mathrm{V}_{\mathrm{k}}\right)$ [15] (método 1) ou parametrizado pelo coeficiente angular da reta [21] (método 2). O primeiro utiliza o preditor tangente e o segundo o preditor secante de ordem zero. Na Fig. 9 pode-se ver a janela principal do programa de fluxo de potência continuado com as suas respectivas opções de comandos:

$\checkmark$ Caso Base: apresenta a curva P-V da barra crítica do sistema escolhido.

$\checkmark$ Executar: possibilita a execução do FPC e a plotagem da curva P-V por completo;

$\checkmark$ Executar por Ponto: permite a execução do FPC e a plotagem da curva $\mathrm{P}-\mathrm{V}$ ponto a ponto. Essa opção é utilizada, p. ex., quando se deseja acompanhar o desempenho do FPC durante o traçado da curva P-V;

$\checkmark$ Plotar Curvas P-Vs: permite plotar a curva P-V de qualquer uma das barras do sistema escolhido. Observase que esta opção só é habilitada após a execução do FPC;

$\checkmark$ Limpar: esta opção possibilita limpar a tela antes que uma nova curva $\mathrm{P}-\mathrm{V}$ seja plotada;

$\checkmark$ Relatório: permite a visualização e impressão dos relatórios e do diagrama unifilar com todas as tensões e ângulos para o PMC;

$\checkmark$ Alterações na Rede: permite alteração de parâmetros ou a retirada de uma linha do sistema escolhido através do diagrama unifilar do sistema, antes da execução de um novo FPC;

$\checkmark$ Contingência Total: simula a retirada individual de cada uma das linhas de transmissão e realiza automaticamente o ranqueamento das dez piores contingências de acordo com a margem de estabilidade;

$\checkmark$ Curva QV: chama a caixa de diálogo para se efetuar o traçado das curvas $\mathrm{Q}-\mathrm{V}$;

$\checkmark$ Análise $Q V$ : chama a caixa de diálogo para efetuar análise Q-V.

Como exemplo é apresentado na Fig. 9 a curva P-V para a barra crítica do sistema IEEE-30. O gráfico foi obtido por meio do FPC considerando a magnitude da tensão da barra 30 como parâmetro da continuação [15] (método 1), partindo do caso base $(\lambda=1)$ e adotando $\sigma=0,2$. Observa-se que para todos os testes realizados, a tolerância adotada para os desbalanços de potência é igual a $10^{-4}$ p.u. Após o traçado da curva $\mathrm{P}-\mathrm{V}$, fornece-se os valores da magnitude $(0,60$ p.u. $)$ e ângulo da tensão $\left(-43,8^{\circ}\right)$ correspondente ao $\operatorname{PMC}(\lambda=1,5410)$, bem como a margem de carregamento $(\mathrm{MC}=0,5410) \mathrm{com}$ relação ao ponto de operação inicialmente escolhido (no caso, $\lambda=1,0)$. Após o traçado da curva, pode-se visualizar ou imprimir, para o PMC em particular, os relatórios e o diagrama unifilar com todas as tensões e ângulos, conforme apresentados nas Figs. 13 e 14.

Já a Fig. 10 apresenta os mesmos resultados da Fig. 9, a diferença é o método do FPC utilizado para o traçado da curva $\mathrm{P}-\mathrm{V}$. Neste caso é o método 2, que se baseia no preditor secante de ordem zero. Os valores do fator de carregamento, 
magnitude e ângulo da tensão no PMC foram $(1,5329,0,574$ e $\left.-45,4^{\circ}\right)$, respectivamente.

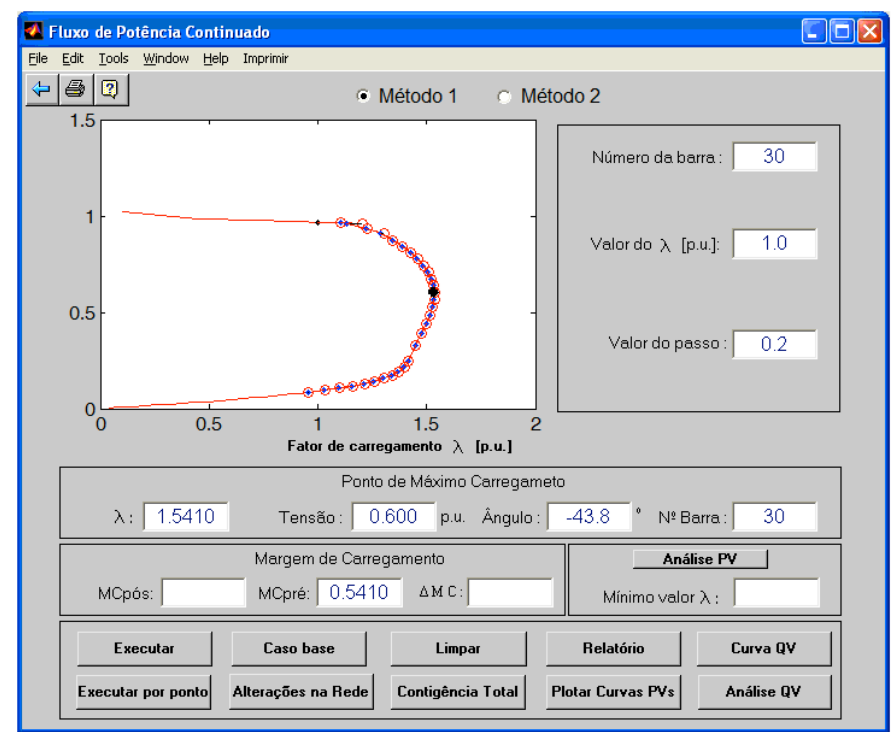

Figura 9 - Tela inicial do programa de fluxo de potência continuado

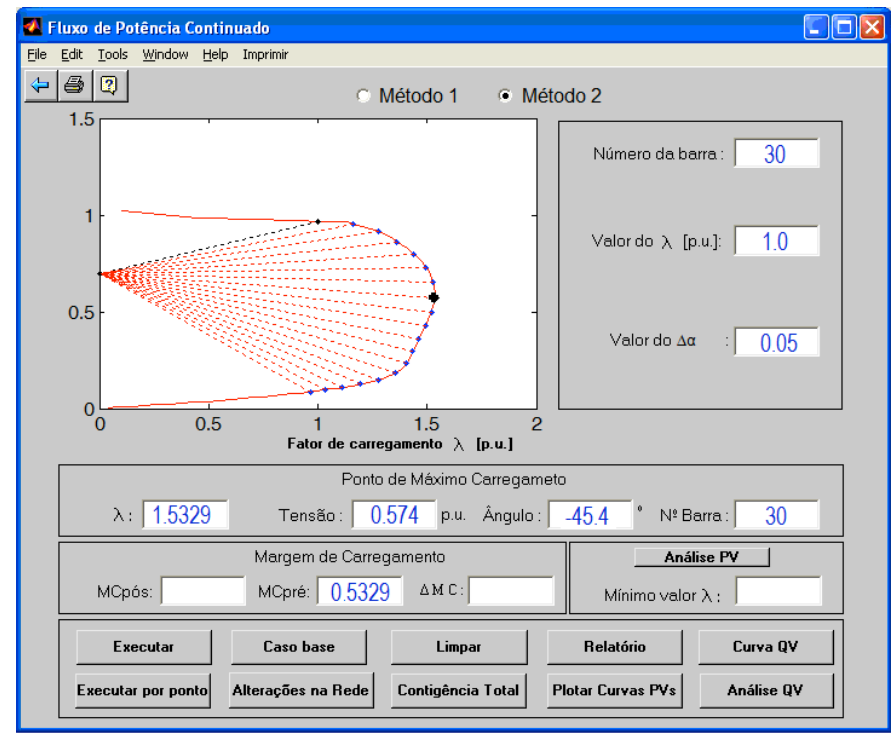

Figura 10 - Caixa de diálogo do FPC apresentando a curva P-V para a barra crítica (barra 30) do sistema IEEE-30 barras.

Caso o usuário deseje ver a curva $\mathrm{P}-\mathrm{V}$ de outra barra do sistema, basta clicar no botão "Plotar curvas PVs" que abrirá uma janela para a escolha da barra (Fig. 11).

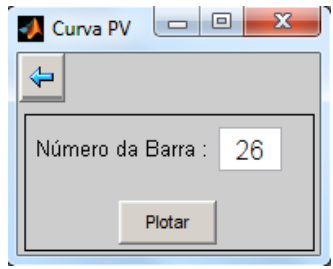

Figura 11: Plotar outra curva P-V do sistema analisado.

A Fig. 12 apresenta a curva P-V da barra escolhida, neste caso a barra 26 do sistema IEEE-30 barras. Na Fig. 13 apresenta-se o relatório do FPC para o sistema IEEE-30 barras, onde se pode ver todos os valores do fator de carregamento $(\lambda)$, magnitude e ângulos das tensões calculados durante o traçado da referida curva P-V da Fig. 9 (método 1), e em particular, para o PMC. A Fig. 14 apresenta o relatório do fluxo de potência em que são mostrados todos os dados de barras tais como magnitude e ângulo da tensão, potência ativa e reativa injetada e consumida e de fluxo nas linhas de transmissão e nos transformadores, para o caso base $(\lambda=1)$.

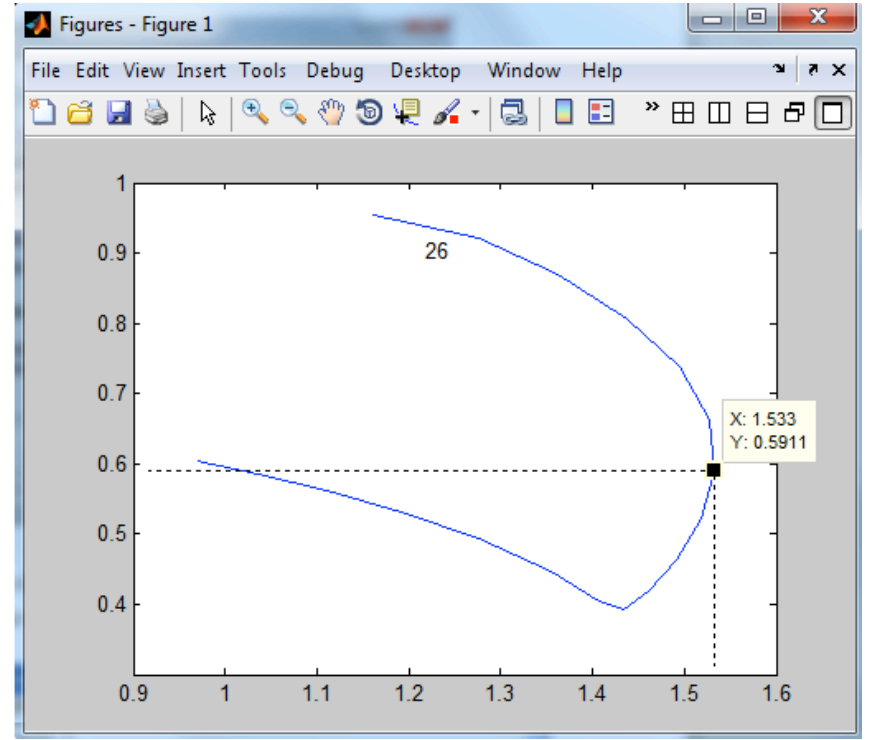

Figura 12: Curva P-V da barra 26 do sistema IEEE-30 barras.

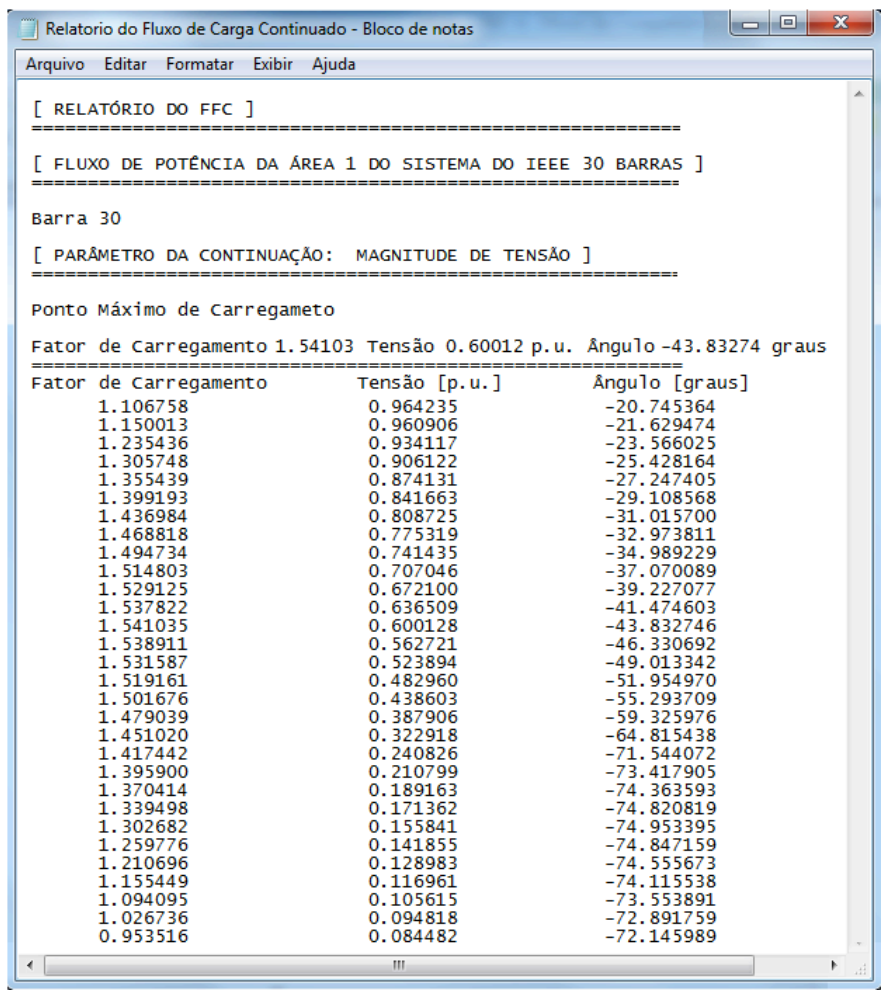

Figura 13 - Relatório do FPC: Valores do carregamento, magnitude e ângulo da tensão para cada ponto da curva P-V do sistema IEEE-30 barras. 


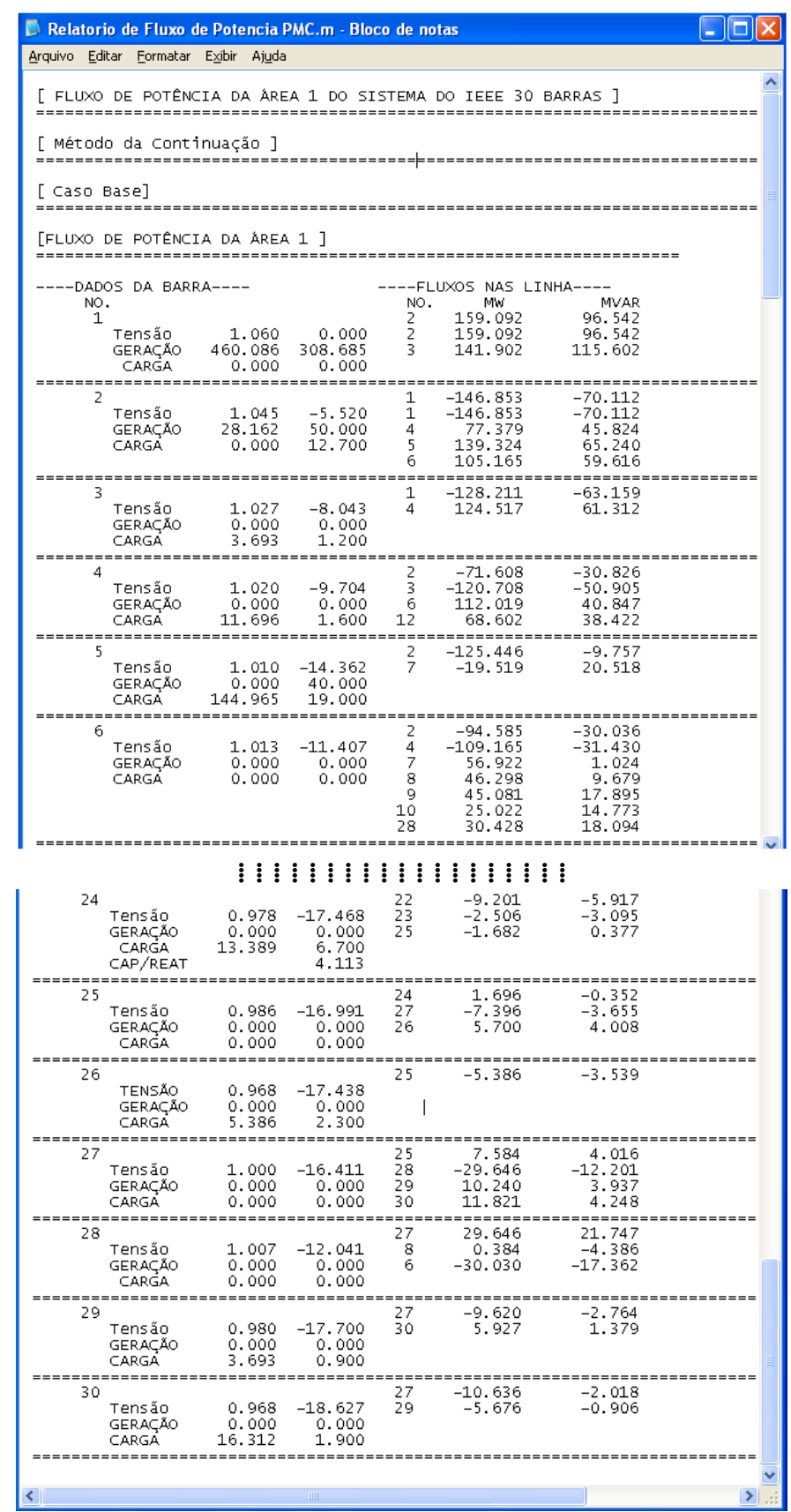

Figura 14 - Relatório do FP para o caso base para todas as barras do sistema IEEE-30 barras.

A Fig. 15(a) e (b) apresenta, respectivamente, o gráfico de barra e de linha do perfil das tensões correspondentes ao caso base e o PMC do sistema IEEE-30 barras, obtidas pelo FPC usando a magnitude de tensão da barra $30\left(\mathrm{~V}_{30}\right)$ como parâmetro da continuação (método 1).

Nas Figs. 16 e 17 apresentam-se os diagramas unifilares do sistema IEEE-30 barras com todos os ângulos e tensões correspondentes ao PMC, também obtidas pelo FPC usando a magnitude de tensão da barra $30\left(\mathrm{~V}_{30}\right)$ como parâmetro da continuação. Cada faixa de tensão é classificada por uma cor, conforme apresenta a tabela de cores, localizada no canto superior esquerdo da janela, o que facilita a identificação visual da barra com maior e menor tensão, bem como da condição crítica em que o sistema se encontra.

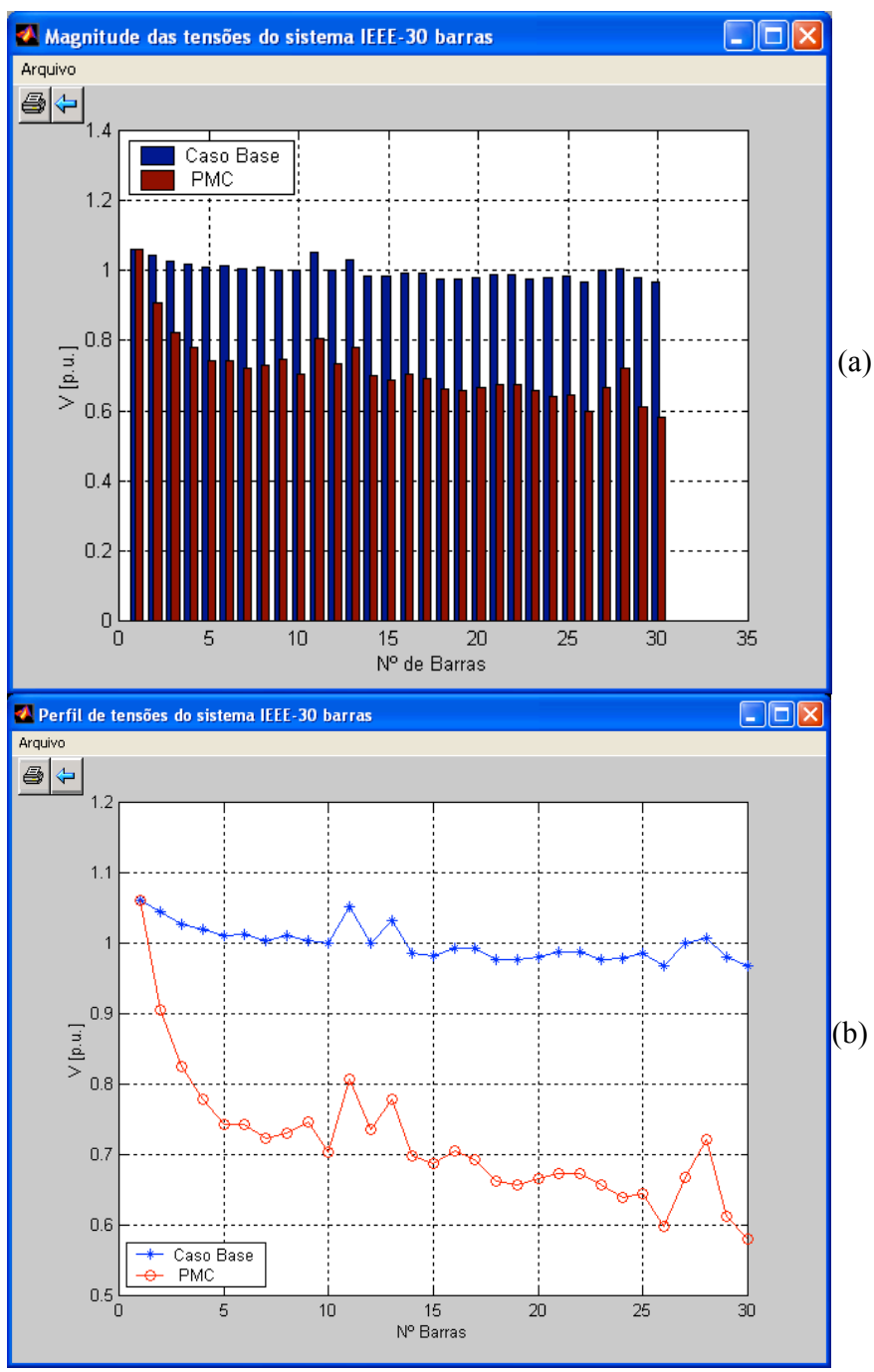

Figura 15 - (a) Perfil de tensão (gráfico de barras no caso base e PMC), (b) Perfil de tensão (gráfico de linhas no caso base e PMC) do sistema IEEE-30 barras.

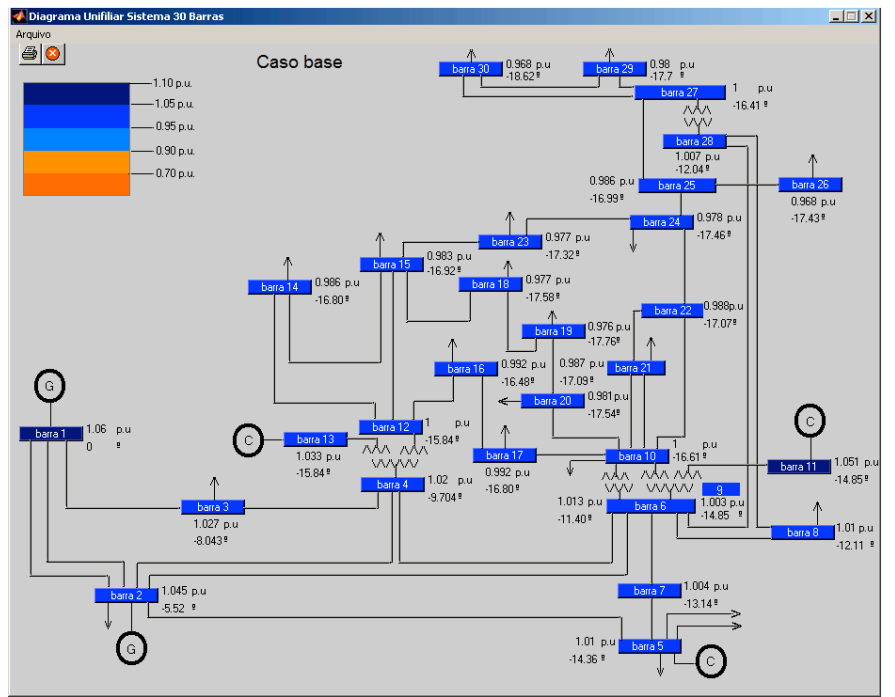

Figura 16 - Diagrama unifilar do sistema IEEE-30 barras com as magnitudes e os ângulos das tensões de barras correspondentes ao caso base, obtidas pelo FPC utilizando $\mathrm{V}_{30}$ como parâmetro da continuação (método 1). 
A Fig. 16 apresenta a faixa de tensão para o caso base, nota-se que praticamente todas as barras apresentam coloração com a tensão na faixa em torno de 1 p.u. Já a Fig. 17 é apresentado a faixa de tensão para o PMC, nota-se a coloração mais avermelhada devido aos níveis de tensão estarem em torno de 0,7 p.u.

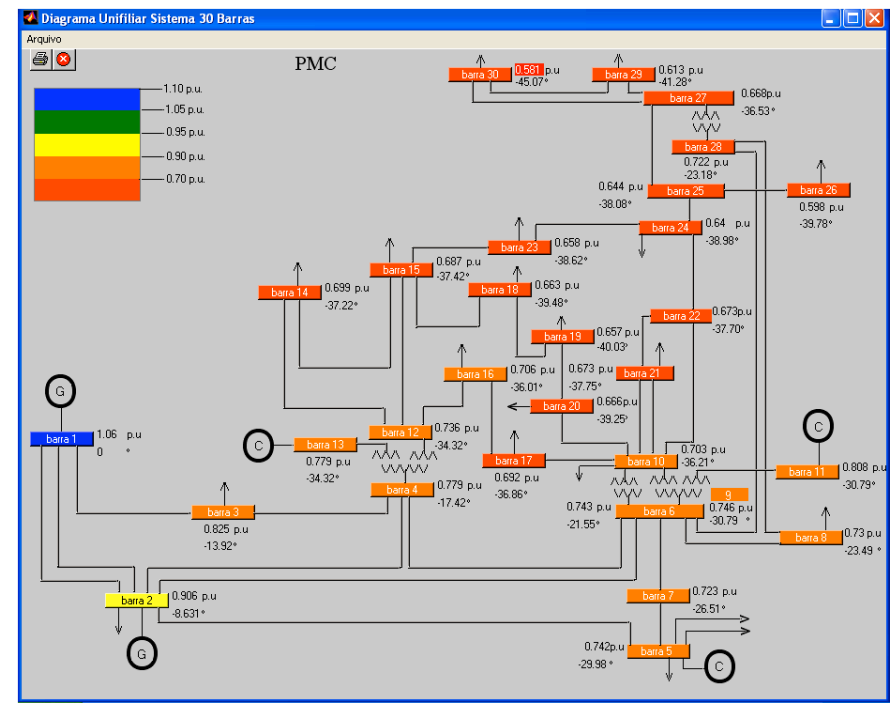

Figura 17 - Diagrama unifilar do sistema IEEE-30 barras com as magnitudes e os ângulos das tensões de barras correspondentes ao PMC, obtidas pelo FPC utilizando $\mathrm{V}_{30}$ como parâmetro da continuação (método 1).

Para analisar contingências por meio da interface gráfica, basta clicar na linha entre as barras escolhidas, como exemplo, a linha entre as barras 2 e 5 do diagrama unifilar apresentado na Fig. 5. Ao clicar, uma nova janela se abre conforme a Fig. 18. Há duas opções, retirar ou consultar a linha selecionada. Se clicar em consultar, uma tela com todos os dados do circuito selecionado aparecerá (Fig. 19). Caso contrário (clicar em retirar), uma nova figura com o diagrama unifilar aparecerá, e neste caso, sem a linha selecionada representando a contingência do sistema (Fig. 20).

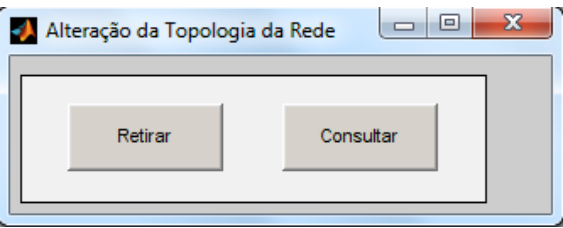

Figura 18 - Tela de consulta ou retirada da linha de transmissão.

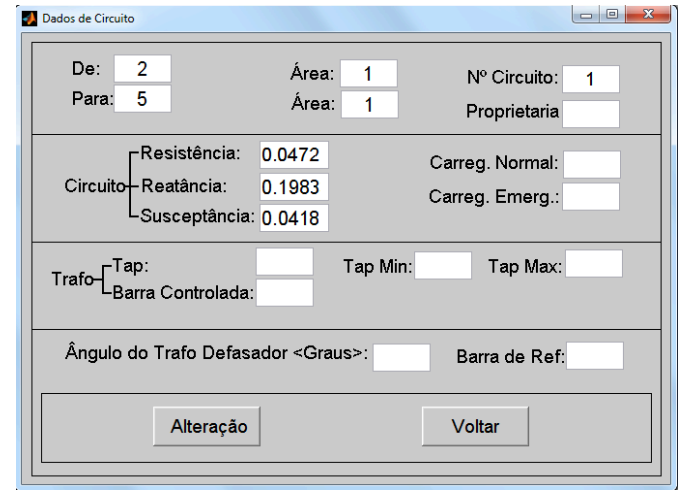

Figura 19 - Dados da linha de transmissão.

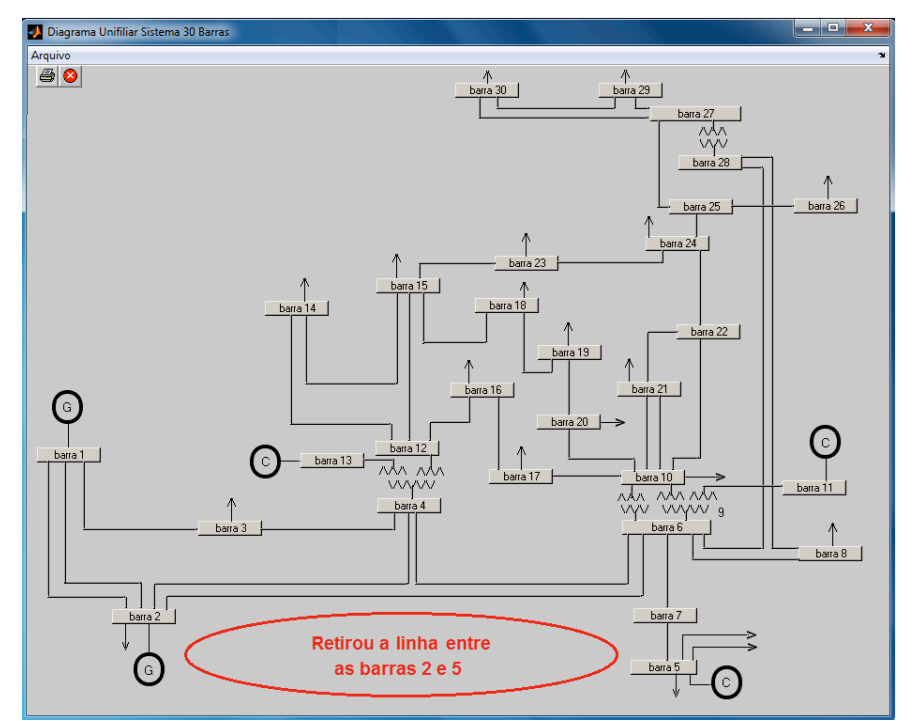

Figura 20 - Diagrama unifilar do sistema IEEE-30 barras sem a linha de transmissão entre as barras 2 e 5 .

Na Fig. 21, é apresentada a janela principal do FPC, porém agora, é utilizada para análise de contingência. Observa-se que duas curvas foram obtidas, uma para o caso base e a outra para a contingência da linha de transmissão situada entre as barras 2 e 5. Os valores dos respectivos PMC foram obtidos $(1,541$ para a curva do caso base e 1,1328 para a curva de contingência), acarretando numa redução da margem de carregamento de 0,4141 aproximadamente. Estes resultados foram obtidos pelo método 2 com passo $\Delta \alpha=0,05$.

A Fig. 22 corresponde às dez piores contingências do sistema IEEE-30 barras, sendo a pior delas localizada entre as barras 1 e 2 .

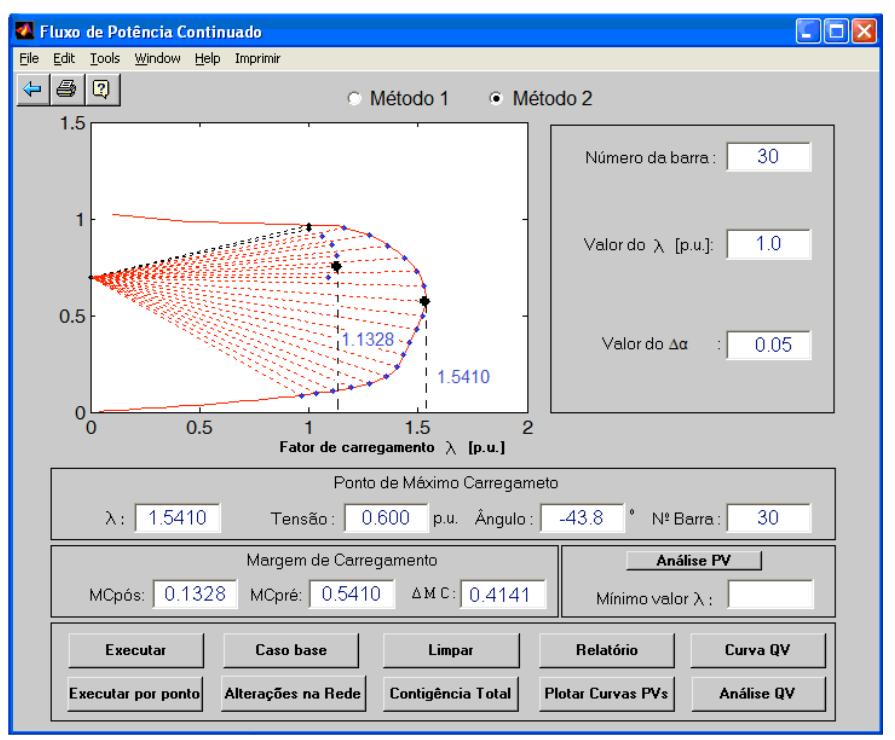

Figura 21 - Janela inicial do programa de fluxo de potência continuado para análise de contingência. 
$\mathrm{O}$ relatório do FPC pode ser visto na Fig. 23, onde se apresentam para cada ponto da curva $\mathrm{P}-\mathrm{V}$, os valores do fator de carregamento, da magnitude e do ângulo da tensão. Os valores do PMC obtidos foram 1,1327, 0,7646 e $-31,867^{\circ}$ respectivamente, obtidos pelo método 2 apresentado na Fig. 21.

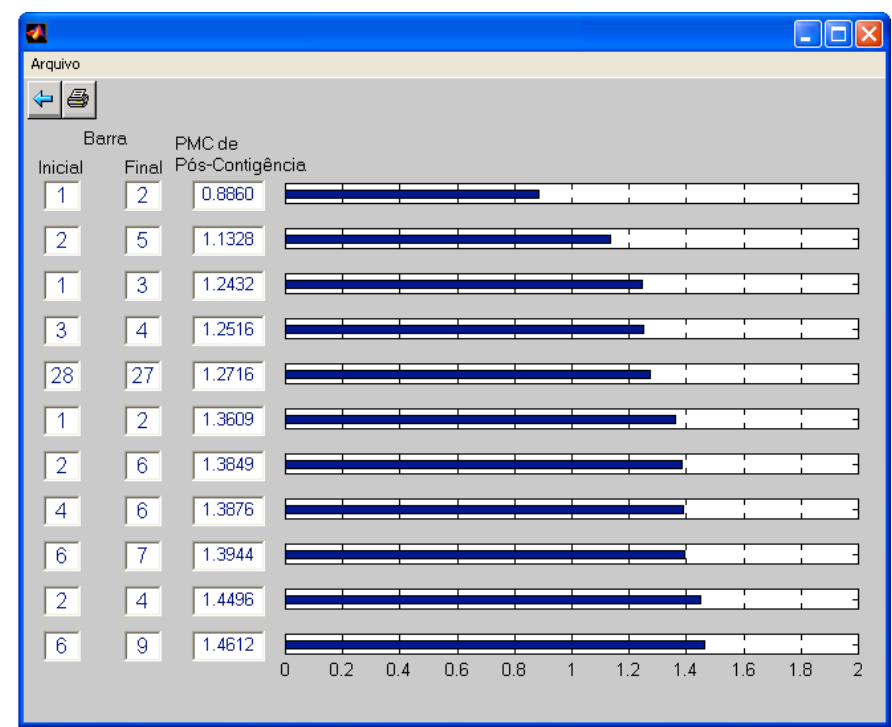

Figura 22 - Dez piores contingências do sistema IEEE-30 barras.

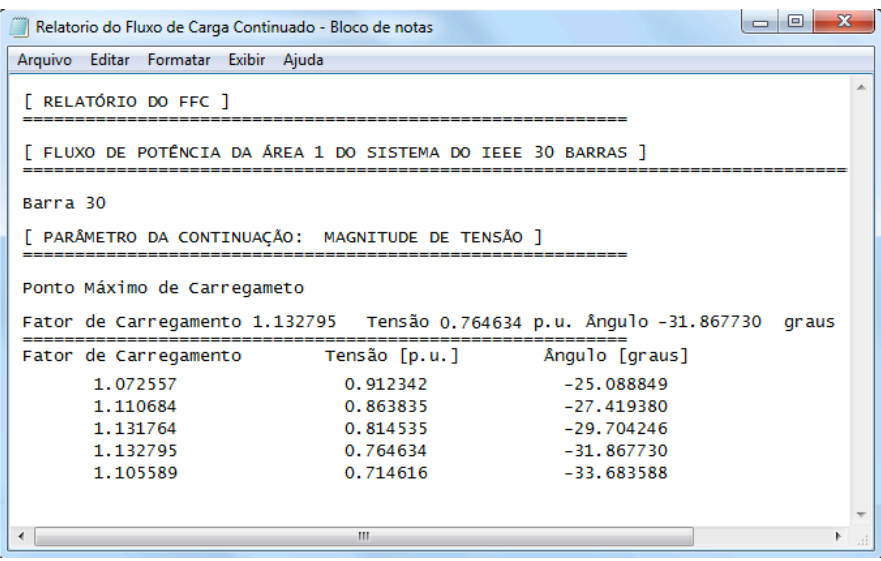

Figura 23 - Relatório do FPC: Valores do carregamento, magnitude e ângulo da tensão para cada ponto da curva P-V de pós-contingência do sistema IEEE30 barras.

Para validar o software desenvolvido, o mesmo foi apresentado aos alunos do curso de engenharia de biossistemas do campus de Tupã. Estes provêm de uma ampla formação em assuntos aqui abordados, com disciplinas de Circuitos Elétricos I e II, Introdução a Ciência da Computação, Programação Orientada a Objetos - UML, Circuitos Digitais, Microcontroladores, Fundamentos de Automação, entre outras [26]. Com isso, a classe foi dividida em dois grupos. Foram abordados vários tópicos como: definição de sistemas elétricos de potência; obtenção do ponto de máximo carregamento; curva $\mathrm{P}-\mathrm{V}$; entre outros, verbalmente e com o uso da lousa, ou seja, de modo convencional. Depois se abordou o mesmo conteúdo com o auxílio do software desenvolvido e aqui apresentado. Verificou-se, em diálogo com os alunos, que o grupo para o qual o conteúdo foi ministrado por intermédio do software, apresentou não só uma maior segurança e melhor entendimento dos assuntos abordados, mas também se mostraram mais interessados a respeito do assunto, reforçando com isso a importância do uso deste tipo de recurso em sala de aula, quando do ensino de novos conceitos.

\section{CONCLUSÃO}

Neste trabalho apresentou-se um programa interativo para a simulação do fluxo de potência continuado. As simulações foram realizadas por meio de uma interface gráfica interativa. A interface gráfica proporciona não só um ambiente mais amigável para o usuário, mas também auxilia e facilita o aprendizado dos estudantes de graduação e pós-graduação em engenharia, para a aquisição de conceitos e no desenvolvimento de programas relacionados com o problema do fluxo de potência. Dois métodos foram apresentados, um que utiliza a técnica de parametrização local e outro que utiliza o coeficiente angular de uma reta localizada no plano de coordenadas formada pelas variáveis fator de carregamento e a magnitude da tensão nodal de uma barra $k$ qualquer. $\mathrm{O}$ software Matlab foi utilizado para programar a interface gráfica e os programas de fluxo de potência e de fluxo de potência continuado. Todas estas características tornam o programa desenvolvido adequado para fins educativos.

\section{AGRADECIMENTOS}

Os autores agradecem a UNESP, Capes e ao CNPq pelo apoio financeiro.

\section{REFERÊNCIAS}

[1] M. Kezunovic, A. Abur, Huang G. The Role of Digital Modeling and Simulation in Power Engineering Education, IEEE Trans. Power Systems, vol. 19, no. 1, p. 64-72, 2004.

[2] A. B. Proto. Interface Gráfica para o Planejamento da Expansão da Transmissão de Energia Elétrica. Dissertação apresentada à UNESP Campus de Ilha Solteira. 102p. 2009.

[3] D. B. R. Góes, G. M. da Silva, D. L. Guedes, O. F. Silva Desenvolvimento de Interface em MATLAB para Aprendizado e Comparação de Métodos Numéricos. XXXV CNMAC - Brazilian Society of Applied and Computational Mathematics. vol. 3, n. 1, 2015.

[4] L. R. A. Gabriel Filho, F. F. Putti, C. P. Cremasco, D. Bordin, M. G. M. Chacur, L. R. A. Gabriel. Software to Assess Beef Cattle Body Mass Through the Fuzzy Body Mass Index. Engenharia Agrícola (Online), v. 36, p. 179-193, 2016.

[5] A. S. B. Teixeira. Desenvolvimento de uma Interface Gráfica para Classificadores de Imagem. Dissertação apresentada ao Instituto Politécnico de Castelo Branco. 74p. 2011.

[6] V. Leal Neto. Desenvolvimento de uma Interface Gráfica de Usuário para Modelos Computacionais de Exposição Externa. Dissertação apresentada à Universidade Federal de Pernambuco. 81p. 2007.

[7] R. T. Gasparini. Desenvolvimento de um Software em Ambiente Matlab para Simulação de Campo Ultrassônico. Dissertação apresentada à UNICAMP. 107p. 2012.

[8] L. H. S. Silva, K. D. V. Mishina, C. T. T. Farias, M. C. Rodrigues, I. S. Ribeiro. Desenvolvimento de uma Interface Gráfica no Ambiente Matlab 
para Avaliação do Risco em Oleodutos On-Shore. VI Congresso Nacional De Engenharia Mecânica. 2010.

[9] A. Bonini Neto, F. F. Putti, R. C. G. Sant'ana, N. A. Bonini Junior. Descriptive Statistics for Data Analysis (Soy Production) via Iterative Program. Brazilian Journal of Biosystems Engineering. v. 6, n. 2, p. 6170, 2012.

[10] FTCT- Força Tarefa Colapso de Tensão. Critérios e metodologias estabelecidos no âmbito da força-tarefa "colapso de tensão" do GTAD/SCEL/GCOI para estudos de estabilidades de tensão nos sistemas interligados Norte/Nordeste, Sul/Sudeste e Norte/Sul Brasileiros, In: Seminário Nacional de Produção e Transmissão de Energia Elétrica SNPTEE, 15. Foz do Iguaçu: Cigré Brasil/Itaipu Binacional, 1999.

[11] WSCC-Reactive Power Reserve Work Group (RRWG). Final report: voltage stability criteria, undervoltage load shedding strategy, and reactive power reserve monitoring methodology, 145 p., Maio 1998.

[12] A. Bonini Neto, E. M. Magalhães, D. A. Alves. Dishonest Newton Method Applied in Continuation Power Flow Through a Geometric Parameterization Technique. Revista IEEE América Latina, v. 14, p. 161170,2016

[13] R. Seydel, "From Equilibrium to Chaos: Pratical Bifurcation and Stability Analisys", 2th. ed., Springer-Verlag: New York, 1994.

[14] A. Bonini Neto, D. A. Alves. Improved geometric parameterization techniques for continuation power flow. IET Generation, Transmission \& Distribution; vol. 4, p. 1349-1359. 2010.

[15] V. Ajjarapu and C.Christy. The Continuation Power Flow: a Tool for Steady State Voltage Stability Analysis. IEEE Trans. on Power Systems, vol. 7, n. 1, February, pp. 416-423, 1992.

[16] E. M. Magalhães, A. Bonini Neto, D. A. Alves. A parameterization technique for the continuation power flow developed from the analysis of power flow curves. Mathematical Problems in Engineering, vol. 2012, p. $1-24,2012$.

[17] H. D. Chiang, A. J. Flueck, K. S. Shah, and N. Balu. "CPFLOW: A practical tool for tracing power systems steady-state stationary behavior due to load and generation variations", IEEE Trans. Power Systems, vol. 10, p. 623-634. 1995.

[18] A. Bonini Neto, E. M. Magalhães, D. A. Alves. Obtaining the Maximum Loading Point of Electric Power Systems using the Decoupled Methods of Newton. Revista IEEE América Latina, v. 14, p. 371-379, 2016.

[19] H. A. Carhullanqui, A. Bonini Neto, D. A. Alves. Continuation Power Flow Considering Area Net Interchange Constraint. International Journal of Electrical Power \& Energy Systems, v. 70, p. 118-126, 2015.

[20] R. R. Matarucco, A. Bonini Neto, D. A. Alves. Assessment of branch outage contingencies using the continuation method. International Journal of Electrical Power \& Energy Systems, v. 55, p. 74-81, 2014.

[21] A. Bonini Neto, D. A. Alves. Técnica de Parametrização Geométrica para o Fluxo de Carga Continuado Baseado nas Variáveis Tensão Nodal e Fator de Carregamento. Controle \& Automação, v.19, p.350 - 366, 2008.

[22] V. Ajjarapu. Computational Techniques for Voltage Stability Assessment and Control. Power Electronics and Power Systems Series, Springer. 2010.

[23] A. Bonini Neto, D. A. Alves. Técnicas de Parametrização Global para o Fluxo de Carga Continuado. Controle \& Automação, v. 21, p. 323-337, 2010.

[24] J. H. Canossa. Um Programa Interativo para Estudos de Fluxo de Potência. Dissertação apresentada à UNESP - Campus de Ilha Solteira, para obtenção do título de Mestre em Engenharia Elétrica. 166p. 2007.

[25] MATHWORKS. Matlab (MATrix LABoratory) Disponível em: $<$ http://www.mathworks.com>. Acesso em 21 de maio de 2015.

[26] Estrutura curricular - Engenharia de Biossistemas. Disponível em: < http://www.tupa.unesp.br/Home/AreaGraduacao/biossistemas/disciplinas _engbiossistemas.pdf $>$
Computação, com ênfase em Arquitetura de Sistemas de Computação e Linux, atuando principalmente nos seguintes temas: fluxo de potência, métodos da continuação e softwares educacionais.

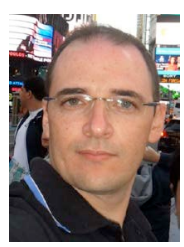

A. Bonini Neto, graduado em Matemática pela Faculdade de Dracena - UNIFADRA, Brasil, em 2002, mestrado e doutorado em Engenharia Elétrica pela Universidade Estadual Paulista - UNESP, campus de Ilha Solteira em 2006 e 2011 respectivamente. Trabalha com pesquisas na área de matemática aplicada na engenharia, técnicas de parametrização no fluxo de carga continuado. Atualmente é professor assistente doutor da Universidade Estadual Paulista - UNESP, campus de Tupã.

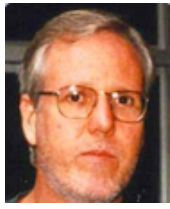

D. A. Alves, graduado em Engenharia Elétrica pela Universidade de São Paulo - USP, Brasil em 1980, mestrado e doutorado em Engenharia Elétrica pela Universidade Campinas - UNICAMP em 1988 e 2000 respectivamente. Trabalha com pesquisas na área de fluxo de carga continuado. Atualmente é professor adjunto da Universidade Estadual Paulista - UNESP, campus de Ilha

Solteira.

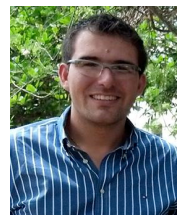

F. F. Putti, graduado em Administração e Agronegócio Universidade Estadual Paulista - UNESP, campus de Tupã, Brasil em 2012, mestrado e doutorado em Agronomia (Irrigação e Drenagem) pela Universidade Estadual Paulista, UNESP, campus de Botucatu, 2014 e 2015 respectivamente. Trabalha com pesquisas na área de modelagem fuzzy. Atualmente é professor assistente doutor da Universidade Estadual Paulista - UNESP, campus de Tupã.



L. R. A. G. Filho, graduado em Matemática, Universidade Estadual Paulista, UNESP, campus de Presidente Prudente, Brasil em 2000, mestrado em matemática pela Universidade de São Paulo, USP em 2004 e doutorado em Agronomia (Energia na Agricultura) pela Universidade Estadual Paulista - UNESP, campus de Botucatu 2007. Trabalha com pesquisas na área de modelagem matemática aplicada em Ciências Agrárias. Atualmente é professor adjunto da Universidade Estadual Paulista - UNESP, campus de Tupã

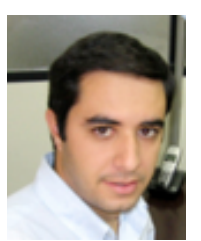

J. Henrique Canossa, graduado em Sistemas de Informação pelo Centro Universitário de Votuporanga (2003) e Mestrado em Engenharia Elétrica pela Universidade Estadual Paulista - UNESP, campus de Ilha Solteira (2007). Tem experiência na área de Ciência da 